Kragujevac Journal of Mathematics

Volume 42(1) (2018), Pages 107-129.

\title{
APPLICATION OF SYMMETRIC BILATERAL BAILEY TRANSFORMATION
}

\author{
MAHESHWAR PATHAK ${ }^{1}$ AND PANKAJ SRIVASTAVA ${ }^{2}$
}

\begin{abstract}
In the present paper, making use of the symmetric bilateral Bailey transformation, we have developed certain relations for basic hypergeometric series and derived their special cases respectively.
\end{abstract}

\section{INTRODUCTION}

W. N. Bailey [27], in 1947 introduced the following transformation formula. If

$$
\beta_{n}=\sum_{r=0}^{n} \alpha_{r} u_{n-r} v_{n+r}, \quad \gamma_{n}=\sum_{r=n}^{\infty} \delta_{r} u_{r-n} v_{r+n}
$$

then

$$
\sum_{n=0}^{\infty} \alpha_{n} \gamma_{n}=\sum_{n=0}^{\infty} \beta_{n} \delta_{n} .
$$

where $\alpha_{r}, \delta_{r}, u_{r}$ and $v_{r}$ are functions of $r$ only, such that the series for $\gamma_{n}$ exists.

This transformation leads to various results which play an important role in number theory and transformation theory of ordinary and basic hypergeometric series both.

Making use of the transformation, Bailey [27,28] developed technique to obtain transformations for both ordinary and basic hypergeometric series and successfully used this transformations to obtain a number of identities of Rogers-Ramanujan type. In 1951 and 1952, L. J. Slater [15,16] derived one hundred and thirty identities of Rogers-Ramanujan type with the help of Bailey transformation formula.

Key words and phrases. Basic hypergeometric series, Bilateral basic hypergeometric series, Symmetric bilateral Bailey transformation.

2010 Mathematics Subject Classification. Primary: 33D15. Secondary: 33D70.

Received: September 12, 2016.

Accepted: December 23, 2016. 
After Bailey and Slater, a number of mathematicians working in the field of ordinary and basic hypergeometric series namely R. P. Agarwal [20], G. E. Andrews [7,8,10], D. M. Bressoud [5], D. M. Bressoud et al. [6], R. Y. Denis et al. [21], C. M. Joshi and Y. Vyas [4], A. Schilling and S. Ole Warnaar [1], S. P. Singh [23], U. B. Singh [24], P. Srivastava [19], A. Verma and V. K. Jain [2,3], S. Ole Warnaar [22] have used Bailey transformation to develop a number of results for both ordinary and basic hypergeometric series, i.e. for ${ }_{2} F_{1},{ }_{3} F_{2},{ }_{4} F_{3},{ }_{5} F_{4}$ etc. and ${ }_{2} \phi_{1},{ }_{3} \phi_{2},{ }_{4} \phi_{3},{ }_{5} \phi_{4}$ etc. with single and multiple bases.

Before the year 1960, few applications of basic hypergeometric series were known. In 1961, N. G. Van Kampen [18] has used basic hypergeometric series to fluctuations in an electric circuit consisting of a condenser and a diode. In 1970, M. Baker and D. D. Coon [17] have used basic hypergeometric series on particle physics. In 1974, G. E. Andrews [9] wrote a paper to uncover applications of basic hypergeometric series in several area of pure and applied mathematics. In [9], G. E. Andrews surveys recent applications of basic hypergeometric series to partitions, number theory, finite vector spaces, combinatorial identities and physics. In the year 1999, V. K. Tuan [25], in 2000, I. Ali and V. K. Tuan [14], and in 2001, V. K. Tuan and M. Z. Nashed [26] have successfully utilized basic hypegeometric series to analytic continuation for functions of complex variable.

In 2007, G. E. Andrews and S. Ole Warnaar [11] have introduced Symmetric and Asymmetric bilateral Bailey transformation formulae and established certain results by making use of the formulae. In the present paper we have developed certain new relations for basic hypergeometric series, using Symmetric bilateral Bailey transformation formula due to G. E. Andrews and S. Ole Warnaar [11].

\section{Definitions and Notations}

We shall use the following $q$-symbols, for more details see [12]. For $|q|<1$

$$
\begin{aligned}
(a ; q)_{n} & = \begin{cases}1, & n=0, \\
(1-a)(1-a q)\left(1-a q^{2}\right) \ldots\left(1-a q^{n-1}\right), & n=1,2, \ldots,\end{cases} \\
(a ; q)_{\infty} & =\prod_{s=0}^{\infty}\left(1-a q^{s}\right), \\
(a ; q)_{-n} & =\frac{(-q / a)^{n}}{(q / a ; q)_{n}} q^{n(n-1) / 2}, \\
\left(a_{1}, a_{2}, \ldots, a_{m} ; q\right)_{n} & =\left(a_{1} ; q\right)_{n}\left(a_{2} ; q\right)_{n} \ldots\left(a_{m} ; q\right)_{n}, \\
\left(a_{1}, a_{2}, \ldots, a_{m} ; q\right)_{\infty} & =\left(a_{1} ; q\right)_{\infty}\left(a_{2} ; q\right)_{\infty} \ldots\left(a_{m} ; q\right)_{\infty} .
\end{aligned}
$$

A generalized basic hypergeometric series with base $q$ is defined as

$$
{ }_{r} \phi_{r-1}\left[\begin{array}{c}
a_{1}, a_{2}, \ldots, a_{r} \\
b_{1}, b_{2}, \ldots, b_{r-1}
\end{array} ; q, z\right]=\sum_{n=0}^{\infty} \frac{\left(a_{1} ; q\right)_{n} \ldots\left(a_{r} ; q\right)_{n} z^{n}}{\left(b_{1} ; q\right)_{n} \ldots\left(b_{r-1} ; q\right)_{n}(q ; q)_{n}} .
$$

if $|q|<1$, series (2.1) converges absolutely for $|z|<1$. 
A generalized bilateral basic hypergeometric series with base $q$ is defined as

$$
{ }_{r} \psi_{r}\left[\begin{array}{c}
a_{1}, a_{2}, \ldots, a_{r} \\
b_{1}, b_{2}, \ldots, b_{r}
\end{array} ; q, z\right]=\sum_{n=-\infty}^{\infty} \frac{\left(a_{1} ; q\right)_{n} \ldots\left(a_{r} ; q\right)_{n}}{\left(b_{1} ; q\right)_{n} \ldots\left(b_{r} ; q\right)_{n}} z^{n}
$$

if $|q|<1$, series $(2.2)$ converges for $\left|\frac{b_{1} \ldots b_{r}}{a_{1} \ldots a_{r}}\right|<|z|<1$.

A truncated basic hypergeometric series with base $q$ is defined as:

$$
{ }_{r} \phi_{r-1}\left[\begin{array}{l}
a_{1}, a_{2}, \ldots, a_{r} \\
b_{1}, b_{2}, \ldots, b_{r-1}
\end{array} ; q, z\right]_{k}=\sum_{n=0}^{k} \frac{\left(a_{1} ; q\right)_{n} \ldots\left(a_{r} ; q\right)_{n} z^{n}}{\left(b_{1} ; q\right)_{n} \ldots\left(b_{r-1} ; q\right)_{n}(q ; q)_{n}} .
$$

The above truncated series is converted into the generalized basic hypergeometric series (2.1), when $|z|<1,|q|<1$, provided that $k \rightarrow \infty$.

A truncated bilateral basic hypergeometric series with base $q$ is defined as:

$$
{ }_{r} \psi_{r}\left[\begin{array}{c}
a_{1}, a_{2}, \ldots, a_{r} \\
b_{1}, b_{2}, \ldots, b_{r}
\end{array} ; q, z\right]_{k}=\sum_{n=-k}^{k} \frac{\left(a_{1} ; q\right)_{n} \ldots\left(a_{r} ; q\right)_{n}}{\left(b_{1} ; q\right)_{n} \ldots\left(b_{r} ; q\right)_{n}} z^{n} .
$$

The above truncated series (2.3) is converted into the generalized bilateral basic hypergeometric series (2.2), when $\left|\frac{b_{1} \ldots b_{r}}{a_{1} \ldots a_{r}}\right|<|z|<1,|q|<1$, provided that $k \rightarrow \infty$.

Symmetric Bilateral Bailey Transformation due to G. E. Andrews and S. Ole Warnaar [11] is defined as follows.

If

$$
\beta_{n}=\sum_{r=-n}^{n} \alpha_{r} u_{n-r} v_{n+r}
$$

and

$$
\gamma_{n}=\sum_{r=|n|}^{\infty} \delta_{r} u_{r-n} v_{r+n}
$$

then

$$
\sum_{n=-\infty}^{\infty} \alpha_{n} \gamma_{n}=\sum_{n=0}^{\infty} \beta_{n} \delta_{n}
$$

where $\alpha_{r}, \delta_{r}, u_{r}$ and $v_{r}$ are any functions of $r$ only, such that the series for $\gamma_{n}$ exists.

To establish the main results following known summation formulae have been used.

(i) $q$-Gauss sum $[12$, II.8, p. 236]

$$
{ }_{2} \phi_{1}\left[\begin{array}{ll}
a, b & \\
c & ; q, c / a b
\end{array}\right]=\frac{(c / a, c / b ; q)_{\infty}}{(c, c / a b ; q)_{\infty}} .
$$


(ii) Ramanujan's sum [12, II.29, p. 239]

$$
{ }_{1} \psi_{1}\left[\begin{array}{cc}
a & \\
& ; q, z \\
b &
\end{array}\right]=\frac{(q, b / a, a z, q / a z ; q)_{\infty}}{(b, q / a, z, b / a z ; q)_{\infty}} .
$$

(iii) Bailey's sum [12, II.31, p. 239]

$$
{ }_{3} \psi_{3}\left[\begin{array}{l}
b, c, d \\
q / b, q / c, q / d
\end{array} ; q, \frac{q}{b c d}\right]=\frac{(q, q / b c, q / b d, q / c d ; q)_{\infty}}{(q / b, q / c, q / d, q / b c d ; q)_{\infty}} .
$$

(iv) Basic bilateral analogue of Dixon's sum [12, II.32, p. 239]

$$
\begin{aligned}
& { }_{4} \psi_{4}\left[\begin{array}{ll}
-q a^{\frac{1}{2}}, b, c, d & \\
-a^{\frac{1}{2}}, a q / b, a q / c, a q / d & q, \frac{q a^{\frac{3}{2}}}{b c d}
\end{array}\right] \\
& =\frac{\left(a q, a q / b c, a q / b d, a q / c d, q a^{\frac{1}{2}} / b, q a^{\frac{1}{2}} / c, q a^{\frac{1}{2}} / d, q, q / a ; q\right)_{\infty}}{\left(a q / b, a q / c, a q / d, q / b, q / c, q / d, q a^{\frac{1}{2}}, q a^{-\frac{1}{2}}, q a^{\frac{3}{2}} / b c d ; q\right)_{\infty}} .
\end{aligned}
$$

(v) Bailey's sum [12, II.33, p. 239]

$$
\begin{aligned}
{ }_{6} \psi_{6}\left[\begin{array}{c}
q a^{\frac{1}{2}},-q a^{\frac{1}{2}}, b, c, d, e \\
a^{\frac{1}{2}},-a^{\frac{1}{2}}, a q / b, a q / c, a q / d, a q / e
\end{array}\right. \\
=\frac{(a q, a q / b c, a q / b d, a q / b e, a q / c d, a q / c e, a q / d e, q, q / a ; q)_{\infty}}{\left(a q / b, a q / c, a q / d, a q / e, q / b, q / c, q / d, q / e, q a^{2} / b c d e ; q\right)_{\infty}} .
\end{aligned}
$$

(vi) Result due to H. S. Shukla [13]

$$
\begin{aligned}
& { }_{8} \psi_{8}\left[\begin{array}{l}
q a^{\frac{1}{2}},-q a^{\frac{1}{2}}, b, c, d, e, f, a q^{2} / f \\
a^{\frac{1}{2}},-a^{\frac{1}{2}}, a q / b, a q / c, a q / d, a q / e, a q / f, f / q \\
b c d e
\end{array}\right] \\
= & \left(1-\frac{(1-b c / a)(1-b d / a)(1-b e / a)}{(1-b q / f)(1-b f / a q)\left(1-b c d e / a^{2}\right)}\right) \frac{(1-f / b q)(1-b f / a q)}{(1-f / a q)(1-f / q)} \\
& \times \frac{(q, a q, q / a, a q / b c, a q / b d, a q / b e, a q / c d, a q / c e, a q / d e ; q)_{\infty}}{\left(a q / b, a q / c, a q / d, a q / e, q / b, q / c, q / d, q / e, a^{2} q / b c d e ; q\right)_{\infty}} .
\end{aligned}
$$

where $\left|a^{2} / b c d e\right|<1$.

\section{MAin Results}

The following results have been established. 
(i)

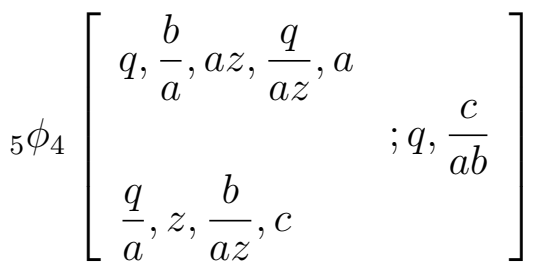

$$
\begin{aligned}
& =\frac{\left(\frac{c}{a}, \frac{c}{b} ; q\right)_{\infty}}{\left(c, \frac{c}{a b} ; q\right)_{\infty}}\left\{{ }_{2} \phi_{1}\left[\begin{array}{ll}
q, a & \\
b & ; q, z
\end{array}\right]+{ }_{2} \phi_{1}\left[\begin{array}{ll}
q, \frac{q}{b} & \\
\frac{q}{a} & ; q, \frac{b}{a z}
\end{array}\right]-1\right\} \\
& +{ }_{2} \phi_{1}\left[\begin{array}{ll}
a, a & ; q, \frac{c z}{a b} \\
c
\end{array}\right]+{ }_{3} \phi_{2}\left[\begin{array}{l}
\frac{q}{b}, a, b \\
\frac{q}{a}, c
\end{array} ; q, \frac{c}{a^{2} z}\right]
\end{aligned}
$$

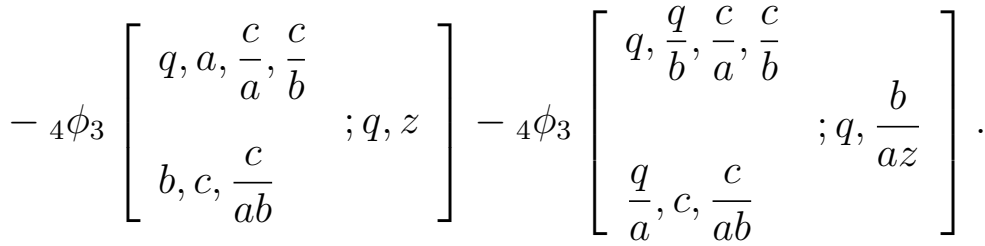

(ii)

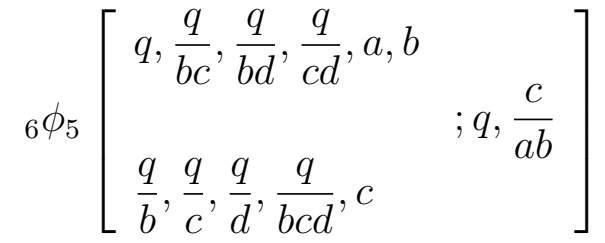

$$
\begin{aligned}
& ={ }_{4} \phi_{3}\left[\begin{array}{ll}
a, b, b, d & ; q, \frac{q}{a b^{2} d} \\
\frac{q}{b}, \frac{q}{c}, \frac{q}{d} &
\end{array}\right]+{ }_{4} \phi_{3}\left[\begin{array}{cc}
a, b, b, d & ; q, \frac{q^{2}}{a b^{2} d} \\
\frac{q}{b}, \frac{q}{c}, \frac{q}{d} &
\end{array}\right] \\
& +\frac{\left(\frac{c}{a}, \frac{c}{b} ; q\right)_{\infty}}{\left(c, \frac{c}{a b} ; q\right)_{\infty}}\left\{{ }_{4} \phi_{3}\left[\begin{array}{c}
q, b, c, d \\
\frac{q}{b}, \frac{q}{c}, \frac{q}{d}
\end{array} ; q, \frac{q}{b c d}\right]+{ }_{4} \phi_{3}\left[\begin{array}{c}
q, b, c, d \\
\frac{q}{b}, \frac{q}{c}, \frac{q}{d}
\end{array} ; q, \frac{q^{2}}{b c d}\right]-1\right\} \\
& \left.-{ }_{5} \phi_{4}\left[\begin{array}{c}
q, b, d, \frac{c}{a}, \frac{c}{b} \\
\frac{q}{b}, \frac{q}{c}, \frac{q}{d}, \frac{c}{a b}
\end{array} ; q, \frac{q}{b c d}\right]-{ }_{5} \phi_{4}\left[\begin{array}{c}
q, b, d, \frac{c}{a}, \frac{c}{b} \\
\frac{q}{b}, \frac{q}{c}, \frac{q}{d}, \frac{c}{a b}
\end{array}\right] q, \frac{q^{2}}{b c d}\right] .
\end{aligned}
$$


(iii)

$$
\begin{aligned}
& \text { (3.3) }{ }_{11} \phi_{10}\left[\begin{array}{c}
a q, \frac{a q}{b c}, \frac{a q}{b d}, \frac{a q}{c d}, \frac{q a^{\frac{1}{2}}}{b}, \frac{q a^{\frac{1}{2}}}{c}, \frac{q a^{\frac{1}{2}}}{d}, q, \frac{q}{a}, a, b \\
\frac{a q}{b}, \frac{a q}{c}, \frac{a q}{d}, \frac{q}{b}, \frac{q}{c}, \frac{q}{d}, q a^{\frac{1}{2}}, q a^{-\frac{1}{2}}, \frac{q a^{\frac{3}{2}}}{b c d}, c
\end{array}\right]
\end{aligned}
$$

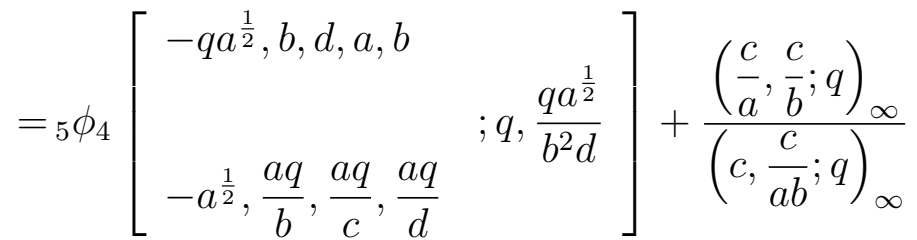

$$
\begin{aligned}
& \left.\times\left\{{ }_{5} \phi_{4}\left[\begin{array}{l}
q,-q a^{\frac{1}{2}}, b, c, d \\
-a^{\frac{1}{2}}, \frac{a q}{b}, \frac{a q}{c}, \frac{a q}{d}
\end{array} ;, \frac{q a^{\frac{3}{2}}}{b c d}\right]+{ }_{5} \phi_{4}\left[\begin{array}{l}
q,-q a^{-\frac{1}{2}}, \frac{b}{a}, \frac{c}{a}, \frac{d}{a} \\
-a^{-\frac{1}{2}}, \frac{q}{b}, \frac{q}{c}, \frac{q}{d}
\end{array}\right] q, \frac{q a^{\frac{3}{2}}}{b c d}\right]-1\right\} \\
& -{ }_{6} \phi_{5}\left[\begin{array}{cc}
q,-q a^{\frac{1}{2}}, b, d, \frac{c}{a}, \frac{c}{b} & \\
-a^{\frac{1}{2}}, \frac{a q}{b}, \frac{a q}{c}, \frac{a q}{d}, \frac{c}{a b} & ; q, \frac{q a^{\frac{3}{2}}}{b c d}
\end{array}\right]
\end{aligned}
$$

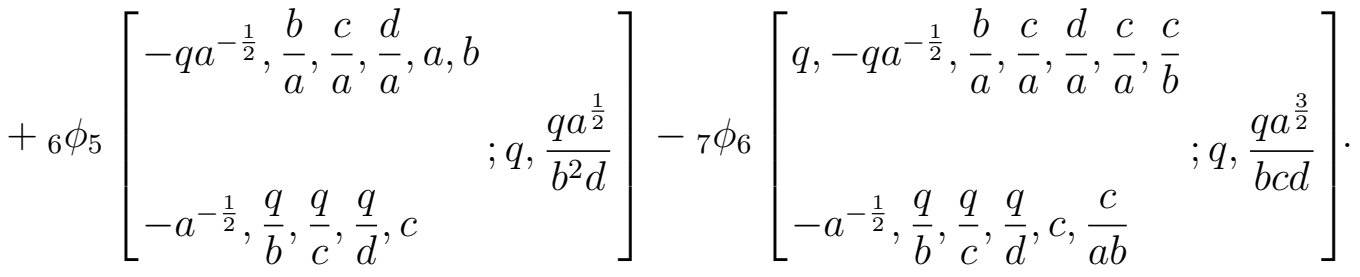

(iv)

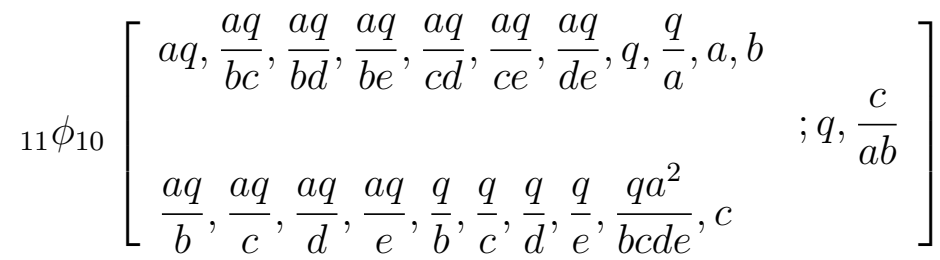

$$
\begin{aligned}
& ={ }_{7} \phi_{6}\left[\begin{array}{cc}
q a^{\frac{1}{2}},-q a^{\frac{1}{2}}, b, d, e, a, b & ; q, \frac{q a}{b^{2} d e} \\
a^{\frac{1}{2}},-a^{\frac{1}{2}}, \frac{a q}{b}, \frac{a q}{c}, \frac{a q}{d}, \frac{a q}{e} &
\end{array}\right]
\end{aligned}
$$




$$
\begin{aligned}
& \left.+\frac{\left(\frac{c}{a}, \frac{c}{b} ; q\right)_{\infty}}{\left(c, \frac{c}{a b} ; q\right)_{\infty} \phi_{6}\left[\begin{array}{l}
q, q a^{\frac{1}{2}},-q a^{\frac{1}{2}}, b, c, d, e \\
a^{\frac{1}{2}},-a^{\frac{1}{2}}, \frac{a q}{b}, \frac{a q}{c}, \frac{a q}{d}, \frac{a q}{e}
\end{array} ; q, \frac{q a^{2}}{b c d e}\right.}\right] \\
& +\frac{\left(\frac{c}{a}, \frac{c}{b} ; q\right)_{\infty}}{\left(c, \frac{c}{a b} ; q\right)_{\infty}}\left\{{ }_{7} \phi_{6}\left[\begin{array}{l}
q, q a^{\frac{1}{2}},-q a^{\frac{1}{2}}, b, c, d, e \\
a^{\frac{1}{2}},-a^{\frac{1}{2}}, \frac{a q}{b}, \frac{a q}{c}, \frac{a q}{d}, \frac{a q}{e}
\end{array} ; q, \frac{q a^{2}}{b c d e}\right]-1\right\} \\
& -{ }_{8} \phi_{7}\left[\begin{array}{cc}
q, q a^{\frac{1}{2}},-q a^{\frac{1}{2}}, b, d, e, \frac{c}{a}, \frac{c}{b} & \\
a^{\frac{1}{2}},-a^{\frac{1}{2}}, \frac{a q}{b}, \frac{a q}{c}, \frac{a q}{d}, \frac{a q}{e}, \frac{c}{a b} & ; q, \frac{q a^{2}}{b c d e}
\end{array}\right] \\
& +{ }_{8} \phi_{7}\left[\begin{array}{ll}
q a^{-\frac{1}{2}},-q a^{-\frac{1}{2}}, \frac{b}{a}, \frac{c}{a}, \frac{d}{a}, \frac{e}{a}, a, b & \\
a^{-\frac{1}{2}},-a^{-\frac{1}{2}}, \frac{q}{b}, \frac{q}{c}, \frac{q}{d}, \frac{q}{e}, c & ; q, \frac{q a}{b^{2} d e}
\end{array}\right]
\end{aligned}
$$

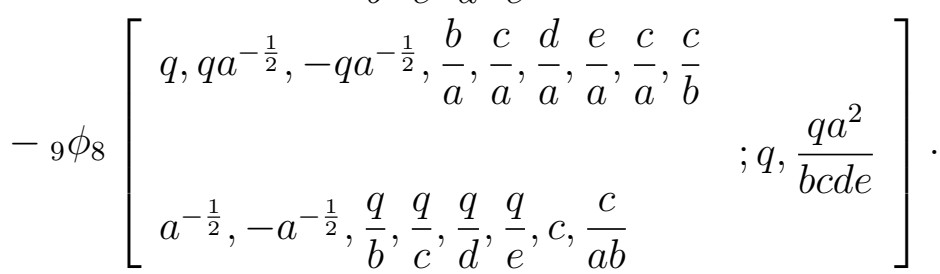

(v)

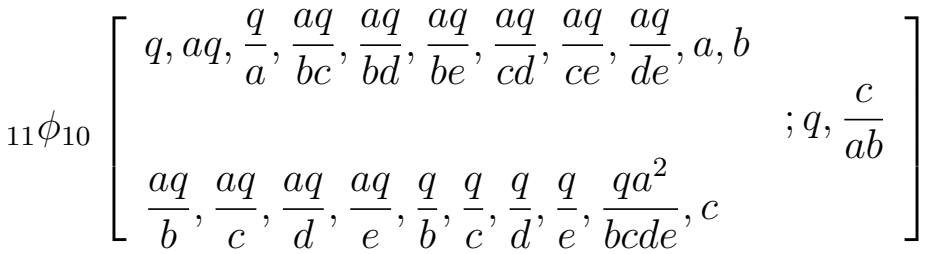

$$
\begin{aligned}
& =\frac{\frac{\left(1-\frac{f}{a q}\right)\left(1-\frac{f}{q}\right)}{\left(1-\frac{f}{b q}\right)\left(1-\frac{b f}{a q}\right)}}{\left(1-\frac{\left(1-\frac{b c}{a}\right)\left(1-\frac{b d}{a}\right)\left(1-\frac{b e}{a}\right)}{\left(1-\frac{b q}{f}\right)\left(1-\frac{b f}{a q}\right)\left(1-\frac{b c d e}{a^{2}}\right)}\right)}
\end{aligned}
$$




$$
\begin{aligned}
& \times\left\{\begin{array}{l}
{ }_{9} \phi_{8}\left[\begin{array}{c}
q a^{\frac{1}{2}},-q a^{\frac{1}{2}}, b, d, e, f, \frac{a q^{2}}{f}, a, b \\
a^{\frac{1}{2}},-a^{\frac{1}{2}}, \frac{a q}{b}, \frac{a q}{c}, \frac{a q}{d}, \frac{a q}{e}, \frac{a q}{f}, \frac{f}{q}
\end{array} ; q \frac{a}{b^{2} d e}\right.
\end{array}\right] \\
& \left.+\frac{\left(\frac{c}{a}, \frac{c}{b} ; q\right)_{\infty}{ }_{9} \phi_{8}}{\left(c, \frac{c}{a b} ; q\right)_{\infty}\left[\begin{array}{l}
q, q a^{\frac{1}{2}},-q a^{\frac{1}{2}}, b, c, d, e, f, \frac{a q^{2}}{f} \\
a^{\frac{1}{2}},-a^{\frac{1}{2}}, \frac{a q}{b}, \frac{a q}{c}, \frac{a q}{d}, \frac{a q}{e}, \frac{a q}{f}, \frac{f}{q}
\end{array} ; q, \frac{a^{2}}{b c d e}\right.}\right] \\
& +\frac{\left(\frac{c}{a}, \frac{c}{b} ; q\right)_{\infty}}{\left(c, \frac{c}{a b} ; q\right)_{\infty}}\left({ }_{9} \phi_{8}\left[\begin{array}{l}
q, q a^{-\frac{1}{2}},-q a^{-\frac{1}{2}}, \frac{b}{a}, \frac{c}{a}, \frac{d}{a}, \frac{e}{a}, \frac{f}{a}, \frac{q^{2}}{f} \\
a^{-\frac{1}{2}},-a^{-\frac{1}{2}}, \frac{q}{b}, \frac{q}{c}, \frac{q}{d}, \frac{q}{e}, \frac{q}{f}, \frac{f}{a q}
\end{array} ; q, \frac{a^{2}}{b c d e f}\right]-1\right)
\end{aligned}
$$

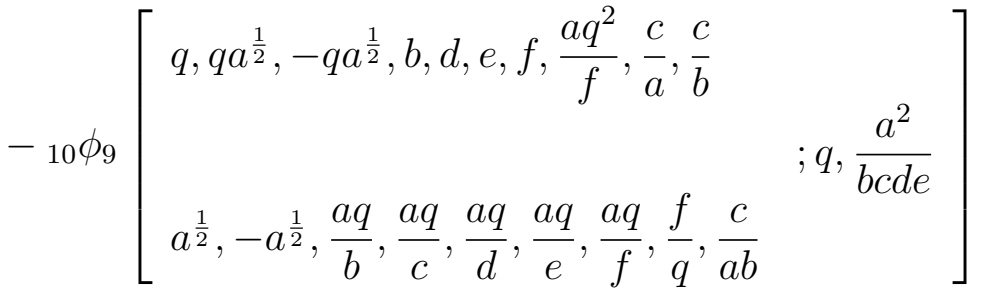

$$
\begin{aligned}
& +{ }_{10} \phi_{9}\left[\begin{array}{ll}
q a^{-\frac{1}{2}},-q a^{-\frac{1}{2}}, \frac{b}{a}, \frac{c}{a}, \frac{d}{a}, \frac{e}{a}, \frac{f}{a}, \frac{q^{2}}{f}, a, b & \\
a^{-\frac{1}{2}},-a^{-\frac{1}{2}}, \frac{q}{b}, \frac{q}{c}, \frac{q}{d}, \frac{q}{e}, \frac{q}{f}, \frac{f}{a q}, c & ; q, \frac{a}{b^{2} d e f}
\end{array}\right]
\end{aligned}
$$

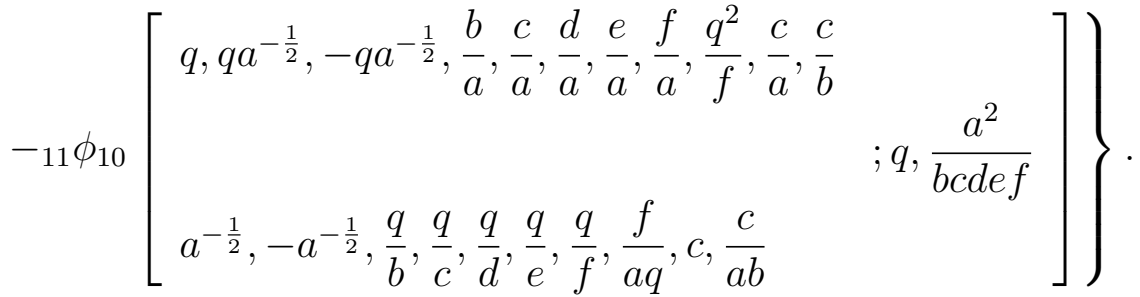

\section{Proof of Main Results}

In this section, we have given the proof of our main results given in $\S 3$. 
Proof of (i). Putting $u_{r}=v_{r}=1$ and $\alpha_{r}=\frac{(a ; q)_{r} z^{r}}{(b ; q)_{r}}$ in (2.4), we get

$$
\beta_{n}=\sum_{r=-n}^{n} \frac{(a ; q)_{r} z^{r}}{(b ; q)_{r}}={ }_{1} \psi_{1}\left[\begin{array}{cc}
a & \\
& ; q, z \\
b &
\end{array}\right]_{n}
$$

making use of the result (2.8), provided $n \rightarrow \infty$, we obtain $\beta_{n}$ as

$$
\beta_{n}=\frac{(q, b / a, a z, q / a z ; q)_{n}}{(b, q / a, z, b / a z ; q)_{n}} .
$$

Putting $u_{r}=v_{r}=1$ in $(2.5)$, we get

$$
\begin{aligned}
\gamma_{n}=\sum_{r=|n|}^{\infty} \delta_{r} & =\delta_{|n|}+\sum_{r=|n|+1}^{\infty} \delta_{r} \\
& =\delta_{|n|}+\sum_{r=0}^{|n|} \delta_{r}+\sum_{r=|n|+1}^{\infty} \delta_{r}-\sum_{r=0}^{|n|} \delta_{r} \\
& =\delta_{|n|}+\sum_{r=0}^{\infty} \delta_{r}-\sum_{r=0}^{|n|} \delta_{r} .
\end{aligned}
$$

Putting

$$
\delta_{r}=\frac{(a, b ; q)_{r}\left(\frac{c}{a b}\right)^{r}}{(q, c ; q)_{r}}
$$

in above equation, we get

$$
\begin{aligned}
\gamma_{n} & =\frac{(a, b ; q)_{|n|}\left(\frac{c}{a b}\right)^{|n|}}{(q, c ; q)_{|n|}}+\sum_{r=0}^{\infty} \frac{(a, b ; q)_{r}\left(\frac{c}{a b}\right)^{r}}{(q, c ; q)_{r}}-\sum_{r=0}^{|n|} \frac{(a, b ; q)_{r}\left(\frac{c}{a b}\right)^{r}}{(q, c ; q)_{r}}, \\
& \left.=\frac{(a, b ; q)_{|n|}\left(\frac{c}{a b}\right)^{|n|}}{(q, c ; q)_{|n|}}+{ }_{2} \phi_{1}\left[\begin{array}{c}
a, b \\
c
\end{array} ; q, \frac{c}{a b}\right]-{ }_{2} \phi_{1}\left[\begin{array}{c}
a, b \\
c
\end{array}\right], \frac{c}{a b}\right]_{|n|}
\end{aligned}
$$

using equation (2.7), provided $n \rightarrow \infty$, we get $\gamma_{n}$ as follows:

$$
\gamma_{n}=\frac{(a, b ; q)_{|n|}\left(\frac{c}{a b}\right)^{|n|}}{(q, c ; q)_{|n|}}+\frac{\left(\frac{c}{a}, \frac{c}{b} ; q\right)_{\infty}}{\left(c, \frac{c}{a b} ; q\right)_{\infty}}-\frac{\left(\frac{c}{a}, \frac{c}{b} ; q\right)_{|n|}}{\left(c, \frac{c}{a b} ; q\right)_{|n|}} .
$$

Putting the values of $\alpha_{n}, \beta_{n}, \gamma_{n}$ and $\delta_{n}$ in (2.6) and after simplification, we obtain result (3.1) as follows. 
L.H.S. of the result (3.1) is obtained as follows

$$
\begin{aligned}
\sum_{n=0}^{\infty} \beta_{n} \delta_{n} & =\sum_{n=0}^{\infty} \frac{\left(q, \frac{b}{a}, a z, \frac{q}{a z} ; q\right)_{n}}{\left(b, \frac{q}{a}, z, \frac{b}{a z} ; q\right)_{n}} \frac{(a, b ; q)_{n}\left(\frac{c}{a b}\right)^{n}}{(q, c ; q)_{n}} \\
& ={ }_{5} \phi_{4}\left[\begin{array}{l}
q, \frac{b}{a}, a z, \frac{q}{a z}, a \\
\frac{q}{a}, z, \frac{b}{a z}, c
\end{array} ;, \frac{c}{a b}\right] .
\end{aligned}
$$

R.H.S. of the result (3.1) is obtained as follows

$$
\begin{aligned}
\sum_{n=-\infty}^{\infty} \alpha_{n} \gamma_{n}= & \sum_{n=-\infty}^{\infty} \frac{(a ; q)_{n} z^{n}}{(b ; q)_{n}}\left\{\frac{(a, b ; q)_{|n|}\left(\frac{c}{a b}\right)^{|n|}}{(q, c ; q)_{|n|}}+\frac{\left(\frac{c}{a}, \frac{c}{b} ; q\right)_{\infty}}{\left(c, \frac{c}{a b} ; q\right)_{\infty}}-\frac{\left(\frac{c}{a}, \frac{c}{b} ; q\right)_{|n|}}{\left(c, \frac{c}{a b} ; q\right)_{|n|}}\right\} \\
= & \sum_{n=0}^{\infty} \frac{(a ; q)_{n} z^{n}}{(b ; q)_{n}}\left\{\frac{(a, b ; q)_{n}\left(\frac{c}{a b}\right)^{n}}{(q, c ; q)_{n}}+\frac{\left(\frac{c}{a}, \frac{c}{b} ; q\right)_{\infty}}{\left(c, \frac{c}{a b} ; q\right)_{\infty}}-\frac{\left(\frac{c}{a}, \frac{c}{b} ; q\right)_{n}}{\left(c, \frac{c}{a b} ; q\right)_{n}}\right\} \\
& +\sum_{n=-\infty}^{-1} \frac{(a ; q)_{n} z^{n}}{(b ; q)_{n}}\left\{\frac{(a, b ; q)_{-n}\left(\frac{c}{a b}\right)^{-n}}{(q, c ; q)_{-n}}+\frac{\left(\frac{c}{a}, \frac{c}{b} ; q\right)_{\infty}}{\left(c, \frac{c}{a b} ; q\right)_{\infty}}-\frac{\left(\frac{c}{a}, \frac{c}{b} ; q\right)_{-n}}{\left(c, \frac{c}{a b} ; q\right)_{-n}}\right\} \\
= & \sum_{n=0}^{\infty} \frac{(a ; q)_{n} z^{n}}{(b ; q)_{n}}\left\{\frac{\left.(a, b ; q)_{n}\left(\frac{c}{a b}\right)^{n}+\frac{c}{a}, \frac{c}{b} ; q\right)_{\infty}}{\left(c, \frac{c}{a b} ; q\right)_{\infty}}, \frac{\left(\frac{c}{a} ; q\right)_{n}}{\left(c, \frac{c}{a b} ; q\right)_{n}}\right\}_{\infty} \\
& +\sum_{n=1}^{\infty} \frac{(a ; q)_{-n} z^{-n}}{(b ; q)_{-n}}\left\{\frac{(a, b ; q)_{n}\left(\frac{c}{a b}\right)^{n}}{(q, c ; q)_{n}}+\frac{\left(\frac{c}{a}, \frac{c}{b} ; q\right)_{\infty}}{\left(c, \frac{c}{a b} ; q\right)_{\infty}}-\frac{\left(\frac{c}{a}, \frac{c}{b} ; q\right)_{n}}{\left(c, \frac{c}{a b} ; q\right)_{n}}\right\}_{\infty}
\end{aligned}
$$

For $n=0$, we get

$$
\begin{aligned}
& \frac{(a ; q)_{-n} z^{-n}}{(b ; q)_{-n}}\left\{\frac{(a, b ; q)_{n}\left(\frac{c}{a b}\right)^{n}}{(q, c ; q)_{n}}+\frac{\left(\frac{c}{a}, \frac{c}{b} ; q\right)_{\infty}}{\left(c, \frac{c}{a b} ; q\right)_{\infty}}-\frac{\left(\frac{c}{a}, \frac{c}{b} ; q\right)_{n}}{\left(c, \frac{c}{a b} ; q\right)_{n}}\right\} \\
= & 1 \times\left\{1+\frac{\left(\frac{c}{a}, \frac{c}{b} ; q\right)_{\infty}}{\left(c, \frac{c}{a b} ; q\right)_{\infty}}-1\right\}
\end{aligned}
$$




$$
=\frac{\left(\frac{c}{a}, \frac{c}{b} ; q\right)_{\infty}}{\left(c, \frac{c}{a b} ; q\right)_{\infty}} .
$$

Therefore, we can write

$$
\begin{aligned}
\sum_{n=-\infty}^{\infty} \alpha_{n} \gamma_{n}= & \sum_{n=0}^{\infty} \frac{(a ; q)_{n} z^{n}}{(b ; q)_{n}}\left\{\frac{(a, b ; q)_{n}\left(\frac{c}{a b}\right)^{n}}{(q, c ; q)_{n}}+\frac{\left(\frac{c}{a}, \frac{c}{b} ; q\right)_{\infty}}{\left(c, \frac{c}{a b} ; q\right)_{\infty}}-\frac{\left(\frac{c}{a}, \frac{c}{b} ; q\right)_{n}}{\left(c, \frac{c}{a b} ; q\right)_{n}}\right\} \\
& +\sum_{n=0}^{\infty} \frac{(a ; q)_{-n} z^{-n}}{(b ; q)_{-n}}\left\{\frac{(a, b ; q)_{n}\left(\frac{c}{a b}\right)^{n}}{(q, c ; q)_{n}}+\frac{\left(\frac{c}{a}, \frac{c}{b} ; q\right)_{\infty}}{\left(c, \frac{c}{a b} ; q\right)_{\infty}}-\frac{\left(\frac{c}{a}, \frac{c}{b} ; q\right)_{n}}{\left(c, \frac{c}{a b} ; q\right)_{n}}\right\} \\
& -\frac{\left(\frac{c}{a}, \frac{c}{b} ; q\right)_{\infty}}{\left(c, \frac{c}{a b} ; q\right)_{\infty}}
\end{aligned}
$$

Since,

$$
\begin{aligned}
\frac{(a ; q)_{-n} z^{-n}}{(b ; q)_{-n}}= & \frac{\frac{\left(-\frac{q}{a}\right)^{n}}{\left(\frac{q}{a} ; q\right)_{n}} q^{\frac{n(n-1)}{2}}}{\frac{\left(-\frac{q}{b}\right)^{n}}{\left(\frac{q}{b} ; q\right)_{n}} q^{\frac{n(n-1)}{2}}} \times \frac{1}{z^{n}} \\
= & \frac{\left(\frac{q}{b} ; q\right)_{n}}{\left(\frac{q}{a} ; q\right)_{n}} \times\left(\frac{b}{a z}\right)^{n}
\end{aligned}
$$

Therefore,

$$
\begin{aligned}
\sum_{n=-\infty}^{\infty} \alpha_{n} \gamma_{n}= & \sum_{n=0}^{\infty} \frac{(a ; q)_{n} z^{n}}{(b ; q)_{n}}\left\{\frac{(a, b ; q)_{n}\left(\frac{c}{a b}\right)^{n}}{(q, c ; q)_{n}}+\frac{\left(\frac{c}{a}, \frac{c}{b} ; q\right)_{\infty}}{\left(c, \frac{c}{a b} ; q\right)_{\infty}}-\frac{\left(\frac{c}{a}, \frac{c}{b} ; q\right)_{n}}{\left(c, \frac{c}{a b} ; q\right)_{n}}\right\} \\
& +\sum_{n=0}^{\infty} \frac{\left(\frac{q}{b} ; q\right)_{n}}{\left(\frac{q}{a} ; q\right)_{n}}\left(\frac{b}{a z}\right)^{n}\left\{\frac{(a, b ; q)_{n}\left(\frac{c}{a b}\right)^{n}}{(q, c ; q)_{n}}+\frac{\left(\frac{c}{a}, \frac{c}{b} ; q\right)_{\infty}}{\left(c, \frac{c}{a b} ; q\right)_{\infty}}-\frac{\left(\frac{c}{a}, \frac{c}{b} ; q\right)_{n}}{\left(c, \frac{c}{a b} ; q\right)_{n}}\right\} \\
& -\frac{\left(\frac{c}{a}, \frac{c}{b} ; q\right)_{\infty}}{\left(c, \frac{c}{a b} ; q\right)_{\infty}}
\end{aligned}
$$




$$
\begin{aligned}
& =\sum_{n=0}^{\infty} \frac{(a ; q)_{n} z^{n}}{(b ; q)_{n}} \frac{(a, b ; q)_{n}\left(\frac{c}{a b}\right)^{n}}{(q, c ; q)_{n}}+\frac{\left(\frac{c}{a}, \frac{c}{b} ; q\right)_{\infty}}{\left(c, \frac{c}{a b} ; q\right)_{\infty}} \sum_{n=0}^{\infty} \frac{(a ; q)_{n} z^{n}}{(b ; q)_{n}} \\
& -\sum_{n=0}^{\infty} \frac{(a ; q)_{n} z^{n}}{(b ; q)_{n}} \frac{\left(\frac{c}{a}, \frac{c}{b} ; q\right)_{n}}{\left(c, \frac{c}{a b} ; q\right)_{n}}+\sum_{n=0}^{\infty} \frac{\left(\frac{q}{b} ; q\right)_{n}\left(\frac{b}{a z}\right)^{n}}{\left(\frac{q}{a} ; q\right)_{n}} \frac{(a, b ; q)_{n}\left(\frac{c}{a b}\right)^{n}}{(q, c ; q)_{n}} \\
& +\frac{\left(\frac{c}{a}, \frac{c}{b} ; q\right)_{\infty}}{\left(c, \frac{c}{a b} ; q\right)_{\infty}} \sum_{n=0}^{\infty} \frac{\left(\frac{q}{b} ; q\right)_{n}\left(\frac{b}{a z}\right)^{n}}{\left(\frac{q}{a} ; q\right)_{n}}-\sum_{n=0}^{\infty} \frac{\left(\frac{q}{b} ; q\right)_{n}\left(\frac{b}{a z}\right)^{n}}{\left(\frac{q}{a} ; q\right)_{n}} \frac{\left(\frac{c}{a}, \frac{c}{b} ; q\right)_{n}}{\left(c, \frac{c}{a b} ; q\right)_{n}} \\
& -\frac{\left(\frac{c}{a}, \frac{c}{b} ; q\right)_{\infty}}{\left(c, \frac{c}{a b} ; q\right)_{\infty}} \\
& ={ }_{2} \phi_{1}\left[\begin{array}{cc}
a, a & ; q, \frac{c z}{a b} \\
c
\end{array}\right]+\frac{\left(\frac{c}{a}, \frac{c}{b} ; q\right)_{\infty}}{\left(c, \frac{c}{a b} ; q\right)_{\infty}} \times{ }_{2} \phi_{1}\left[\begin{array}{ll}
q, a & \\
b & ; q, z
\end{array}\right]
\end{aligned}
$$

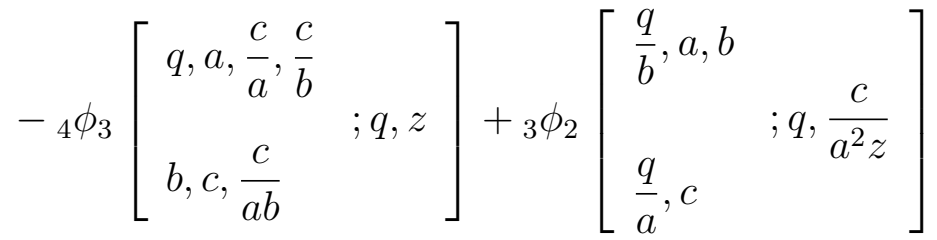

$$
\begin{aligned}
& \left.+\frac{\left(\frac{c}{a}, \frac{c}{b} ; q\right)_{\infty}}{\left(c, \frac{c}{a b} ; q\right)_{\infty}}{ }_{2} \phi_{1}\left[\begin{array}{c}
q, \frac{q}{b} \\
\frac{q}{a}
\end{array} ; q, \frac{b}{a z}\right]-{ }_{4} \phi_{3}\left[\begin{array}{c}
q, \frac{q}{b}, \frac{c}{a}, \frac{c}{b} \\
\frac{q}{a}, c, \frac{c}{a b}
\end{array}\right] ;, \frac{b}{a z}\right] \\
& -\frac{\left(\frac{c}{a}, \frac{c}{b} ; q\right)_{\infty}}{\left(c, \frac{c}{a b} ; q\right)_{\infty}} \\
& =\frac{\left(\frac{c}{a}, \frac{c}{b} ; q\right)_{\infty}}{\left(c, \frac{c}{a b} ; q\right)_{\infty}}\left\{{ }_{2} \phi_{1}\left[\begin{array}{ll}
q, a & \\
b & ; q, z
\end{array}\right]+{ }_{2} \phi_{1}\left[\begin{array}{ll}
q, \frac{q}{b} & \\
\frac{q}{a} & ; q, \frac{b}{a z}
\end{array}\right]-1\right\}
\end{aligned}
$$




$$
\begin{aligned}
& +{ }_{2} \phi_{1}\left[\begin{array}{ll}
a, a & ; q, \frac{c z}{a b} \\
c
\end{array}\right]+{ }_{3} \phi_{2}\left[\begin{array}{ll}
\frac{q}{b}, a, b & ; q, \frac{c}{a^{2} z} \\
\frac{q}{a}, c &
\end{array}\right] \\
& \left.-{ }_{4} \phi_{3}\left[\begin{array}{c}
q, a, \frac{c}{a}, \frac{c}{b} \\
b, c, \frac{c}{a b}
\end{array} ; q, z\right]-{ }_{4} \phi_{3}\left[\begin{array}{c}
q, \frac{q}{b}, \frac{c}{a}, \frac{c}{b} \\
\frac{q}{a}, c, \frac{c}{a b}
\end{array}\right] q, \frac{b}{a z}\right] .
\end{aligned}
$$

From equation (2.6), we have

$$
\sum_{n=0}^{\infty} \beta_{n} \delta_{n}=\sum_{n=-\infty}^{\infty} \alpha_{n} \gamma_{n} .
$$

Hence, the result (3.1) is given as follows

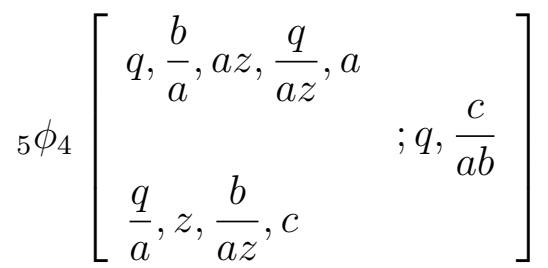

$$
\begin{aligned}
& =\frac{\left(\frac{c}{a}, \frac{c}{b} ; q\right)_{\infty}}{\left(c, \frac{c}{a b} ; q\right)_{\infty}}\left\{{ }_{2} \phi_{1}\left[\begin{array}{ll}
q, a & \\
b & ; q, z
\end{array}\right]+{ }_{2} \phi_{1}\left[\begin{array}{ll}
q, \frac{q}{b} & \\
\frac{q}{a} & ; q, \frac{b}{a z}
\end{array}\right]-1\right\} \\
& +{ }_{2} \phi_{1}\left[\begin{array}{ll}
a, a & ; q, \frac{c z}{a b} \\
c
\end{array}\right]+{ }_{3} \phi_{2}\left[\begin{array}{l}
\frac{q}{b}, a, b \\
\frac{q}{a}, c
\end{array} ; q, \frac{c}{a^{2} z}\right] \\
& \left.-{ }_{4} \phi_{3}\left[\begin{array}{c}
q, a, \frac{c}{a}, \frac{c}{b} \\
b, c, \frac{c}{a b}
\end{array} ; q, z\right]-{ }_{4} \phi_{3}\left[\begin{array}{c}
q, \frac{q}{b}, \frac{c}{a}, \frac{c}{b} \\
\frac{q}{a}, c, \frac{c}{a b}
\end{array}\right] q, \frac{b}{a z}\right] \text {. }
\end{aligned}
$$

Proof of (ii). Putting $u_{r}=v_{r}=1$ and $\alpha_{r}=\frac{(b, c, d ; q)_{r}(q / b c d)^{r}}{(q / b, q / c, q / d ; q)_{r}}$ in (2.4), we get

$$
\beta_{n}=\sum_{r=-n}^{n} \frac{(b, c, d ; q)_{r}\left(\frac{q}{b c d}\right)^{r}}{\left(\frac{q}{b}, \frac{q}{c}, \frac{q}{d} ; q\right)_{r}}={ }_{3} \psi_{3}\left[\begin{array}{l}
b, c, d \\
\frac{q}{b}, \frac{q}{c}, \frac{q}{d}
\end{array} ; q, \frac{q}{b c d}\right]_{n}
$$


using result (2.9), provided $n \rightarrow \infty$, we get $\beta_{n}$ as follows:

$$
\beta_{n}=\frac{\left(q, \frac{q}{b c}, \frac{q}{b d}, \frac{q}{c d} ; q\right)_{n}}{\left(\frac{q}{b}, \frac{q}{c}, \frac{q}{d}, \frac{q}{b c d} ; q\right)_{n}}
$$

Putting the values of $\alpha_{n}, \beta_{n}$ and values of $\delta_{n}, \gamma_{n}$ from (4.1) and (4.2) in (2.6) and after simplification, we obtain result (3.2) as follows.

L.H.S. of the result (3.2) is obtained as

$$
\begin{aligned}
\sum_{n=0}^{\infty} \beta_{n} \delta_{n} & =\sum_{n=0}^{\infty} \frac{\left(q, \frac{q}{b c}, \frac{q}{b d}, \frac{q}{c d} ; q\right)_{n}}{\left(\frac{q}{b}, \frac{q}{c}, \frac{q}{d}, \frac{q}{b c d} ; q\right)_{n}} \frac{(a, b ; q)_{n}\left(\frac{c}{a b}\right)^{n}}{(q, c ; q)_{n}} \\
& ={ }_{6} \phi_{5}\left[\begin{array}{l}
q, \frac{q}{b c}, \frac{q}{b d}, \frac{q}{c d}, a, b \\
\frac{q}{b}, \frac{q}{c}, \frac{q}{d}, \frac{q}{b c d}, c
\end{array} ; q, \frac{c}{a b}\right]
\end{aligned}
$$

R.H.S. of the result (3.2) is obtained as

$$
\begin{aligned}
\sum_{n=-\infty}^{\infty} \alpha_{n} \gamma_{n}= & \sum_{n=-\infty}^{\infty} \frac{(b, c, d ; q)_{n}\left(\frac{q}{b c d}\right)^{n}}{\left(\frac{q}{b}, \frac{q}{c}, \frac{q}{d} ; q\right)_{n}} \\
& \times\left\{\frac{(a, b ; q)_{|n|}\left(\frac{c}{a b}\right)^{|n|}}{(q, c ; q)_{|n|}}+\frac{\left(\frac{c}{a}, \frac{c}{b} ; q\right)_{\infty}}{\left(c, \frac{c}{a b} ; q\right)_{\infty}}-\frac{\left(\frac{c}{a}, \frac{c}{b} ; q\right)_{|n|}}{\left(c, \frac{c}{a b} ; q\right)_{|n|}}\right\} \\
= & \sum_{n=0}^{\infty} \frac{(b, c, d ; q)_{n}\left(\frac{q}{b c d}\right)^{n}}{\left(\frac{q}{b}, \frac{q}{c}, \frac{q}{d} ; q\right)_{n}} \\
& \times\left\{\frac{\left.(a, b ; q)_{n}\left(\frac{c}{a b}\right)^{n}+\frac{\left(\frac{c}{a}, \frac{c}{b} ; q\right)_{\infty}}{(q, c ; q)_{n}}-\frac{\left(\frac{c}{a}, \frac{c}{b} ; q\right)_{n}}{\left(c, \frac{c}{a b} ; q\right)_{n}}\right\}_{\infty}}{(c, q)^{n}}\right. \\
& +\sum_{n=-\infty}^{-1} \frac{(b, c, d ; q)_{n}\left(\frac{q}{b c d}\right)^{n}}{\left(\frac{q}{b}, \frac{q}{c}, \frac{q}{d} ; q\right)_{n}} \\
& \times\left\{\frac{(a, b ; q)_{-n}\left(\frac{c}{a b}\right)^{-n}}{(q, c ; q)_{-n}}+\frac{\left(\frac{c}{a}, \frac{c}{b} ; q\right)_{\infty}}{\left(c, \frac{c}{a b} ; q\right)_{\infty}}-\frac{\left(\frac{c}{a}, \frac{c}{b} ; q\right)_{-n}}{\left(c, \frac{c}{a b} ; q\right)_{-n}}\right\}
\end{aligned}
$$




$$
\begin{aligned}
= & \sum_{n=0}^{\infty} \frac{(b, c, d ; q)_{n}\left(\frac{q}{b c d}\right)^{n}}{\left(\frac{q}{b}, \frac{q}{c}, \frac{q}{d} ; q\right)_{n}} \\
& \times\left\{\frac{(a, b ; q)_{n}\left(\frac{c}{a b}\right)^{n}}{(q, c ; q)_{n}}+\frac{\left(\frac{c}{a}, \frac{c}{b} ; q\right)_{\infty}}{\left(c, \frac{c}{a b} ; q\right)_{\infty}}-\frac{\left(\frac{c}{a}, \frac{c}{b} ; q\right)_{n}}{\left(c, \frac{c}{a b} ; q\right)_{n}}\right\} \\
& +\sum_{n=1}^{\infty} \frac{(b, c, d ; q)_{-n}\left(\frac{q}{b c d}\right)^{-n}}{\left(\frac{q}{b}, \frac{q}{c}, \frac{q}{d} ; q\right)}-n \\
& \times\left\{\frac{(a, b ; q)_{n}\left(\frac{c}{a b}\right)^{n}}{(q, c ; q)_{n}}+\frac{\left(\frac{c}{a}, \frac{c}{b} ; q\right)_{\infty}}{\left(c, \frac{c}{a b} ; q\right)_{\infty}}-\frac{\left(\frac{c}{a}, \frac{c}{b} ; q\right)_{n}}{\left(c, \frac{c}{a b} ; q\right)_{n}}\right\} .
\end{aligned}
$$

For $n=0$, we get

$$
\begin{aligned}
& \frac{(b, c, d ; q)_{-n}\left(\frac{q}{b c d}\right)^{-n}}{\left(\frac{q}{b}, \frac{q}{c}, \frac{q}{d} ; q\right)_{-n}}\left\{\frac{(a, b ; q)_{n}\left(\frac{c}{a b}\right)^{n}}{(q, c ; q)_{n}}+\frac{\left(\frac{c}{a}, \frac{c}{b} ; q\right)_{\infty}}{\left(c, \frac{c}{a b} ; q\right)_{\infty}}-\frac{\left(\frac{c}{a}, \frac{c}{b} ; q\right)_{n}}{\left(c, \frac{c}{a b} ; q\right)_{n}}\right\} \\
= & 1 \times\left\{1+\frac{\left(\frac{c}{a}, \frac{c}{b} ; q\right)_{\infty}}{\left(c, \frac{c}{a b} ; q\right)_{\infty}}-1\right\}=\frac{\left(\frac{c}{a}, \frac{c}{b} ; q\right)_{\infty}}{\left(c, \frac{c}{a b} ; q\right)_{\infty}}
\end{aligned}
$$

Therefore, we can write

$$
\begin{aligned}
\sum_{n=-\infty}^{\infty} \alpha_{n} \gamma_{n}= & \sum_{n=0}^{\infty} \frac{(b, c, d ; q)_{n}\left(\frac{q}{b c d}\right)^{n}}{\left(\frac{q}{b}, \frac{q}{c}, \frac{q}{d} ; q\right)_{n}} \\
& \times\left\{\frac{(a, b ; q)_{n}\left(\frac{c}{a b}\right)^{n}}{(q, c ; q)_{n}}+\frac{\left(\frac{c}{a}, \frac{c}{b} ; q\right)_{\infty}}{\left(c, \frac{c}{a b} ; q\right)_{\infty}}-\frac{\left(\frac{c}{a}, \frac{c}{b} ; q\right)_{n}}{\left(c, \frac{c}{a b} ; q\right)_{n}}\right\} \\
& +\sum_{n=0}^{\infty} \frac{(b, c, d ; q)_{-n}\left(\frac{q}{b c d}\right)^{-n}}{\left(\frac{q}{b}, \frac{q}{c}, \frac{q}{d} ; q\right)_{-n}} \\
& \times\left\{\frac{(a, b ; q)_{n}\left(\frac{c}{a b}\right)^{n}}{(q, c ; q)_{n}}+\frac{\left(\frac{c}{a}, \frac{c}{b} ; q\right)_{\infty}}{\left(c, \frac{c}{a b} ; q\right)_{\infty}}-\frac{\left(\frac{c}{a}, \frac{c}{b} ; q\right)_{n}}{\left(c, \frac{c}{a b} ; q\right)_{n}}\right\}-\frac{\left(\frac{c}{a}, \frac{c}{b} ; q\right)_{\infty}}{\left(c, \frac{c}{a b} ; q\right)_{\infty}} .
\end{aligned}
$$


Since,

$$
\begin{aligned}
& \frac{(b, c, d ; q)_{-n}\left(\frac{q}{b c d}\right)^{-n}}{\left(\frac{q}{b}, \frac{q}{c}, \frac{q}{d} ; q\right)_{-n}} \\
= & \frac{(b ; q)_{-n}(c ; q)_{-n}(d ; q)_{-n}\left(\frac{q}{b c d}\right)^{-n}}{\left(\frac{q}{b} ; q\right)_{-n}\left(\frac{q}{c} ; q\right)_{-n}\left(\frac{q}{d} ; q\right)_{-n}} \\
= & \frac{\left(-\frac{q}{b}\right)^{n}}{\left(\frac{q}{b} ; q\right)_{n} q^{\frac{n(n-1)}{2}} \frac{\left(-\frac{q}{c}\right)^{n}}{\left(\frac{q}{c} ; q\right)_{n}} q^{\frac{n(n-1)}{2}} \frac{\left(-\frac{q}{d}\right)^{n}}{\left(\frac{q}{d} ; q\right)_{n}} q^{\frac{n(n-1)}{2}}}\left(\frac{b c d}{q}\right)^{n} \\
= & \frac{(b ; q)_{n}(c ; q)_{n}(d ; q)_{n}}{\left(\frac{q(n-1)}{2} ; q\right)_{n}\left(\frac{q}{c} ; q\right)_{n}}\left(\frac{(-c)^{n}}{(c ; q)_{n}} q^{\frac{n(n-1)}{2}} \frac{(-d)^{n}}{(d ; q)_{n}} q^{\frac{n(n-1)}{2}}\right)^{n} \\
= & \frac{(b, c, d ; q)_{n}}{\left(\frac{q}{b}, \frac{q}{c}, \frac{q}{d} ; q\right)_{n}}\left(\frac{q^{2}}{b c d}\right)^{n} .
\end{aligned}
$$

Therefore,

$$
\begin{aligned}
& \sum_{n=-\infty}^{\infty} \alpha_{n} \gamma_{n} \\
= & \sum_{n=0}^{\infty} \frac{(b, c, d ; q)_{n}\left(\frac{q}{b c d}\right)^{n}}{\left(\frac{q}{b}, \frac{q}{c}, \frac{q}{d} ; q\right)_{n}}\left\{\frac{(a, b ; q)_{n}\left(\frac{c}{a b}\right)^{n}}{(q, c ; q)_{n}}+\frac{\left(\frac{c}{a}, \frac{c}{b} ; q\right)_{\infty}}{\left(c, \frac{c}{a b} ; q\right)_{\infty}}-\frac{\left(\frac{c}{a}, \frac{c}{b} ; q\right)_{n}}{\left(c, \frac{c}{a b} ; q\right)_{n}}\right\} \\
& +\sum_{n=0}^{\infty} \frac{(b, c, d ; q)_{n}\left(\frac{q^{2}}{b c d}\right)^{n}}{\left(\frac{q}{b}, \frac{q}{c}, \frac{q}{d} ; q\right)_{n}}\left\{\frac{(a, b ; q)_{n}\left(\frac{c}{a b}\right)^{n}}{(q, c ; q)_{n}}+\frac{\left(\frac{c}{a}, \frac{c}{b} ; q\right)_{\infty}}{\left(c, \frac{c}{a b} ; q\right)_{\infty}}-\frac{\left(\frac{c}{a}, \frac{c}{b} ; q\right)_{n}}{\left(c, \frac{c}{a b} ; q\right)_{n}}\right\} \\
& -\frac{\left(\frac{c}{a}, \frac{c}{b} ; q\right)_{\infty}}{\left(c, \frac{c}{a b} ; q\right)_{\infty}} \\
= & \sum_{n=0}^{\infty} \frac{(b, c, d ; q)_{n}\left(\frac{q}{b c d}\right)^{n}}{\left(\frac{q}{b}, \frac{q}{c}, \frac{q}{d} ; q\right)_{n}} \frac{(a, b ; q)_{n}\left(\frac{c}{a b}\right)^{n}}{(q, c ; q)_{n}}+\frac{\left(\frac{c}{a}, \frac{c}{b} ; q\right)_{\infty}}{\left(c, \frac{c}{a b} ; q\right)_{\infty}} \sum_{n=0}^{\infty} \frac{(b, c, d ; q)_{n}\left(\frac{q}{b c d}\right)^{n}}{\left(\frac{q}{b}, \frac{q}{c}, \frac{q}{d} ; q\right)_{n}}
\end{aligned}
$$




$$
\begin{aligned}
& -\sum_{n=0}^{\infty} \frac{(b, c, d ; q)_{n}\left(\frac{q}{b c d}\right)^{n}}{\left(\frac{q}{b}, \frac{q}{c}, \frac{q}{d} ; q\right)_{n}} \frac{\left(\frac{c}{a}, \frac{c}{b} ; q\right)_{n}}{\left(c, \frac{c}{a b} ; q\right)_{n}}+\sum_{n=0}^{\infty} \frac{(b, c, d ; q)_{n}\left(\frac{q^{2}}{b c d}\right)^{n}}{\left(\frac{q}{b}, \frac{q}{c}, \frac{q}{d} ; q\right)_{n}} \frac{(a, b ; q)_{n}\left(\frac{c}{a b}\right)^{n}}{(q, c ; q)_{n}} \\
& +\frac{\left(\frac{c}{a}, \frac{c}{b} ; q\right)_{\infty}}{\left(c, \frac{c}{a b} ; q\right)_{\infty}} \sum_{n=0}^{\infty} \frac{(b, c, d ; q)_{n}\left(\frac{q^{2}}{b c d}\right)^{n}}{\left(\frac{q}{b}, \frac{q}{c}, \frac{q}{d} ; q\right)_{n}}-\sum_{n=0}^{\infty} \frac{(b, c, d ; q)_{n}\left(\frac{q^{2}}{b c d}\right)^{n}}{\left(\frac{q}{b}, \frac{q}{c}, \frac{q}{d} ; q\right)_{n}} \frac{\left(\frac{c}{a}, \frac{c}{b} ; q\right)_{n}}{\left(c, \frac{c}{a b} ; q\right)_{n}} \\
& -\frac{\left(\frac{c}{a}, \frac{c}{b} ; q\right)_{\infty}}{\left(c, \frac{c}{a b} ; q\right)_{\infty}} \\
& ={ }_{4} \phi_{3}\left[\begin{array}{l}
a, b, b, d \\
\frac{q}{b}, \frac{q}{c}, \frac{q}{d}
\end{array} ; q, \frac{q}{a b^{2} d}\right]+\frac{\left(\frac{c}{a}, \frac{c}{b} ; q\right)_{\infty}}{\left(c, \frac{c}{a b} ; q\right)_{\infty} \phi_{3}}\left[\begin{array}{c}
q, b, c, d \\
\frac{q}{b}, \frac{q}{c}, \frac{q}{d}
\end{array} ; q, \frac{q}{b c d}\right] \\
& -{ }_{5} \phi_{4}\left[\begin{array}{c}
q, b, d, \frac{c}{a}, \frac{c}{b} \\
\frac{q}{b}, \frac{q}{c}, \frac{q}{d}, \frac{c}{a b}
\end{array} ; q, \frac{q}{b c d}\right]+{ }_{4} \phi_{3}\left[\begin{array}{ll}
a, b, b, d & ; q, \frac{q^{2}}{a b^{2} d} \\
\frac{q}{b}, \frac{q}{c}, \frac{q}{d} &
\end{array}\right] \\
& +\frac{\left(\frac{c}{a}, \frac{c}{b} ; q\right)_{\infty}}{\left(c, \frac{c}{a b} ; q\right)_{\infty}} \phi_{3}\left[\begin{array}{c}
q, b, c, d \\
\frac{q}{b}, \frac{q}{c}, \frac{q}{d}
\end{array} ; q, \frac{q^{2}}{b c d}\right]-{ }_{5} \phi_{4}\left[\begin{array}{c}
q, b, d, \frac{c}{a}, \frac{c}{b} \\
\frac{q}{b}, \frac{q}{c}, \frac{q}{d}, \frac{c}{a b}
\end{array} ; q, \frac{q^{2}}{b c d}\right] \\
& -\frac{\left(\frac{c}{a}, \frac{c}{b} ; q\right)_{\infty}}{\left(c, \frac{c}{a b} ; q\right)_{\infty}}
\end{aligned}
$$

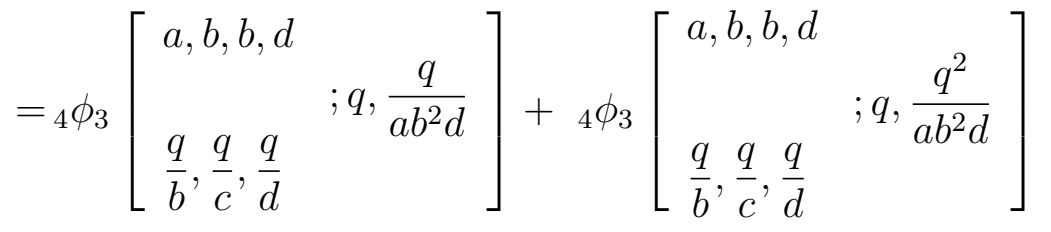

$$
\begin{aligned}
& +\frac{\left(\frac{c}{a}, \frac{c}{b} ; q\right)_{\infty}}{\left(c, \frac{c}{a b} ; q\right)_{\infty}}\left\{{ }_{4} \phi_{3}\left[\begin{array}{c}
q, b, c, d \\
\frac{q}{b}, \frac{q}{c}, \frac{q}{d}
\end{array} ; q, \frac{q}{b c d}\right]+{ }_{4} \phi_{3}\left[\begin{array}{c}
q, b, c, d \\
\frac{q}{b}, \frac{q}{c}, \frac{q}{d}
\end{array} ; q, \frac{q^{2}}{b c d}\right]-1\right\}
\end{aligned}
$$




$$
\left.-{ }_{5} \phi_{4}\left[\begin{array}{c}
q, b, d, \frac{c}{a}, \frac{c}{b} \\
\frac{q}{b}, \frac{q}{c}, \frac{q}{d}, \frac{c}{a b}
\end{array} ; q, \frac{q}{b c d}\right]-{ }_{5} \phi_{4}\left[\begin{array}{c}
q, b, d, \frac{c}{a}, \frac{c}{b} \\
\frac{q}{b}, \frac{q}{c}, \frac{q}{d}, \frac{c}{a b}
\end{array}\right] ; q, \frac{q^{2}}{b c d}\right] .
$$

From equation (2.6), we have

$$
\sum_{n=0}^{\infty} \beta_{n} \delta_{n}=\sum_{n=-\infty}^{\infty} \alpha_{n} \gamma_{n}
$$

Hence, the result (3.2) is given as follows

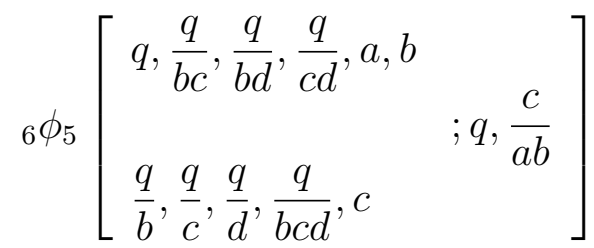

$$
\begin{aligned}
& ={ }_{4} \phi_{3}\left[\begin{array}{c}
a, b, b, d \\
\frac{q}{b}, \frac{q}{c}, \frac{q}{d}
\end{array} ; q, \frac{q}{a b^{2} d}\right]+{ }_{4} \phi_{3}\left[\begin{array}{l}
a, b, b, d \\
\frac{q}{b}, \frac{q}{c}, \frac{q}{d}
\end{array} ; q, \frac{q^{2}}{a b^{2} d}\right] \\
& +\frac{\left(\frac{c}{a}, \frac{c}{b} ; q\right)_{\infty}}{\left(c, \frac{c}{a b} ; q\right)_{\infty}}\left\{{ }_{4} \phi_{3}\left[\begin{array}{c}
q, b, c, d \\
\frac{q}{b}, \frac{q}{c}, \frac{q}{d}
\end{array} ; q, \frac{q}{b c d}\right]+{ }_{4} \phi_{3}\left[\begin{array}{l}
q, b, c, d \\
\frac{q}{b}, \frac{q}{c}, \frac{q}{d}
\end{array} ; q, \frac{q^{2}}{b c d}\right]-1\right\} \\
& \left.-{ }_{5} \phi_{4}\left[\begin{array}{c}
q, b, d, \frac{c}{a}, \frac{c}{b} \\
\frac{q}{b}, \frac{q}{c}, \frac{q}{d}, \frac{c}{a b}
\end{array} ; q, \frac{q}{b c d}\right]-{ }_{5} \phi_{4}\left[\begin{array}{c}
q, b, d, \frac{c}{a}, \frac{c}{b} \\
\frac{q}{b}, \frac{q}{c}, \frac{q}{d}, \frac{c}{a b}
\end{array}\right] ; q, \frac{q^{2}}{b c d}\right] .
\end{aligned}
$$

Following the similar procedure as in the proof of main results (i) and (ii), one can easily derive the main results (iii), (iv) and (v). which are given as follows.

Proof of (iii). Putting $u_{r}=v_{r}=1$ and $\alpha_{r}=\frac{\left(-q a^{\frac{1}{2}}, b, c, d ; q\right)_{r}\left(q a^{\frac{3}{2}} / b c d\right)^{r}}{\left(-a^{\frac{1}{2}}, a q / b, a q / c, a q / d ; q\right)_{r}}$ in $(2.4)$ and making use of the result (2.10), provided $n \rightarrow \infty$, we get $\beta_{n}$, which is as follows:

$$
\beta_{n}=\frac{\left(a q, \frac{a q}{b c}, \frac{a q}{b d}, \frac{a q}{c d}, \frac{q a^{\frac{1}{2}}}{b}, \frac{q a^{\frac{1}{2}}}{c}, \frac{q a^{\frac{1}{2}}}{d}, q, \frac{q}{a} ; q\right)_{n}}{\left(\frac{a q}{b}, \frac{a q}{c}, \frac{a q}{d}, \frac{q}{b}, \frac{q}{c}, \frac{q}{d}, q a^{\frac{1}{2}}, q a^{-\frac{1}{2}}, \frac{q a^{\frac{3}{2}}}{b c d} ; q\right)_{n}}
$$


Putting the values of $\alpha_{n}, \beta_{n}$ and values of $\delta_{n}, \gamma_{n}$ from (4.1) and (4.2) in (2.6) and after simplification, we obtain result (3.3).

Proof of (iv). Putting $u_{r}=v_{r}=1$ and $\alpha_{r}=\frac{\left(q a^{\frac{1}{2}},-q a^{\frac{1}{2}}, b, c, d, e ; q\right)_{r}\left(q a^{2} / b c d e\right)^{r}}{\left(a^{\frac{1}{2}},-a^{\frac{1}{2}}, a q / b, a q / c, a q / d, a q / e ; q\right)_{r}}$ in (2.4) and making use of the result (2.11), provided $n \rightarrow \infty$, we get $\beta_{n}$, which is as follows:

$$
\beta_{n}=\frac{\left(a q, \frac{a q}{b c}, \frac{a q}{b d}, \frac{a q}{b e}, \frac{a q}{c d}, \frac{a q}{c e}, \frac{a q}{d e}, q, \frac{q}{a} ; q\right)_{n}}{\left(\frac{a q}{b}, \frac{a q}{c}, \frac{a q}{d}, \frac{a q}{e}, \frac{q}{b}, \frac{q}{c}, \frac{q}{d}, \frac{q}{e}, \frac{q a^{2}}{b c d e} ; q\right)_{n}}
$$

Putting the values of $\alpha_{n}, \beta_{n}$ and values of $\delta_{n}, \gamma_{n}$ from (4.1) and (4.2) in (2.6) and after simplification, we obtain result (3.4).

Proof of (v). Putting $u_{r}=v_{r}=1$ and

$$
\alpha_{r}=\frac{\left(q a^{\frac{1}{2}},-q a^{\frac{1}{2}}, b, c, d, e, f, a q^{2} / f ; q\right)_{r}\left(a^{2} / b c d e\right)^{r}}{\left(a^{\frac{1}{2}},-a^{\frac{1}{2}}, a q / b, a q / c, a q / d, a q / e, a q / f, f / q ; q\right)_{r}}
$$

in (2.4) and making use of the result (2.12), provided $n \rightarrow \infty$, we get $\beta_{n}$, which is as follows

$$
\begin{aligned}
\beta_{n}= & \left(1-\frac{\left(1-\frac{b c}{a}\right)\left(1-\frac{b d}{a}\right)\left(1-\frac{b e}{a}\right)}{\left(1-\frac{b q}{f}\right)\left(1-\frac{b f}{a q}\right)\left(1-\frac{b c d e}{a^{2}}\right)}\right) \frac{\left(1-\frac{f}{b q}\right)\left(1-\frac{b f}{a q}\right)}{\left(1-\frac{f}{a q}\right)\left(1-\frac{f}{q}\right)} \\
& \times \frac{\left(q, a q, \frac{q}{a}, \frac{a q}{b c}, \frac{a q}{b d}, \frac{a q}{b e}, \frac{a q}{c d}, \frac{a q}{c e}, \frac{a q}{d e} ; q\right)_{n}}{\left(\frac{a q}{b}, \frac{a q}{c}, \frac{a q}{d}, \frac{a q}{e}, \frac{q}{b}, \frac{q}{c}, \frac{q}{d}, \frac{q}{e}, \frac{q a^{2}}{b c d e} ; q\right)_{n}}
\end{aligned}
$$

Putting the values of $\alpha_{n}, \beta_{n}$ and values of $\delta_{n}, \gamma_{n}$ from (4.1) and (4.2) in (2.6) and after simplification, we obtain result (3.5).

\section{Special Cases}

Certain interesting results have been developed from the main results as special cases, which are as follows:

(i) Putting $b=q$ in result (3.1), special case is as follows

$$
{ }_{3} \phi_{2}\left[\begin{array}{ll}
q, a z, a & \\
z, c & ; q, \frac{c}{a q}
\end{array}\right]=\frac{\left(\frac{c}{a}, \frac{c}{q} ; q\right)_{\infty}}{\left(c, \frac{c}{a q} ; q\right)_{\infty}} \phi_{0}\left[\begin{array}{cc}
a & \\
- & ; q, z
\end{array}\right]
$$




$$
+{ }_{2} \phi_{1}\left[\begin{array}{cc}
a, a & ; q, \frac{c z}{a q} \\
c
\end{array}\right]-{ }_{3} \phi_{2}\left[\begin{array}{c}
a, \frac{c}{a}, \frac{c}{q} \\
c, \frac{c}{a q}
\end{array} ; q, z\right]
$$

(ii) Putting $a=c$ in result (3.2), special case is as follows

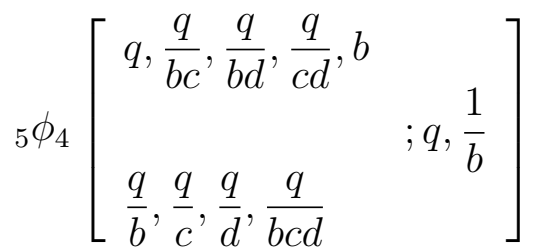

$$
\begin{aligned}
& ={ }_{4} \phi_{3}\left[\begin{array}{c}
b, b, c, d \\
\frac{q}{b}, \frac{q}{c}, \frac{q}{d}
\end{array} ; q, \frac{q}{b^{2} c d}\right]+{ }_{4} \phi_{3}\left[\begin{array}{cc}
b, b, c, d & \\
\frac{q}{b}, \frac{q}{c}, \frac{q}{d} & ; q, \frac{q^{2}}{b^{2} c d}
\end{array}\right]-2 .
\end{aligned}
$$

(iii) Putting $a=c$ in the result (3.3), special case is as follows

$$
{ }_{5} \phi_{4}\left[\begin{array}{l}
c q, \frac{c q}{b d}, \frac{q c^{\frac{1}{2}}}{b}, \frac{q c^{\frac{1}{2}}}{d}, b \\
\frac{c q}{b}, \frac{c q}{d}, q c^{\frac{1}{2}}, \frac{q c^{\frac{1}{2}}}{b d}
\end{array} ;, \frac{1}{b}\right]={ }_{5} \phi_{4}\left[\begin{array}{ll}
-q c^{\frac{1}{2}}, b, b, c, d & \\
-c^{\frac{1}{2}}, \frac{c q}{b}, q, \frac{c q}{d} & ; q, \frac{q c^{\frac{1}{2}}}{b^{2} d}
\end{array}\right]-1 .
$$

(iv) Putting $a=c$ in the result (3.4), special case is as follows

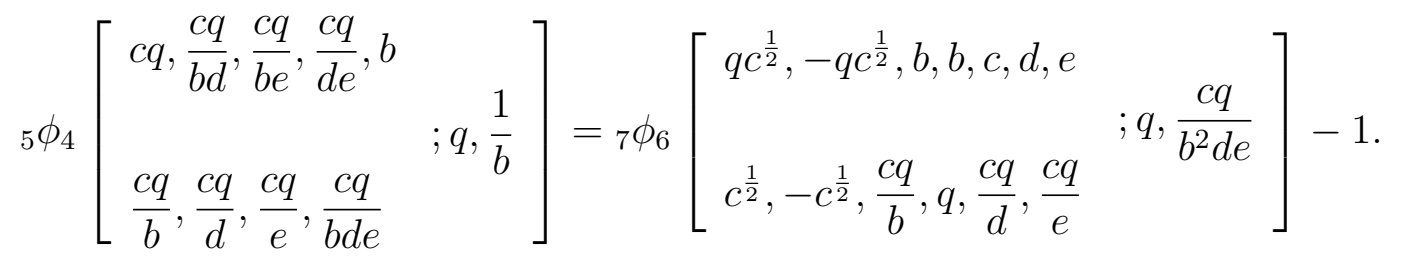

(v) Putting $a=c$ in the result (3.5), special case is as follows

$$
{ }_{5} \phi_{4}\left[\begin{array}{c}
c q, \frac{c q}{b d}, \frac{c q}{b e}, \frac{c q}{d e}, b \\
\frac{c q}{b}, \frac{c q}{d}, \frac{c q}{e}, \frac{c q}{b d e}
\end{array} ; q, \frac{1}{b}\right]
$$




$$
\begin{aligned}
& =\frac{\frac{\left(1-\frac{f}{c q}\right)\left(1-\frac{f}{q}\right)}{\left(1-\frac{f}{b q}\right)\left(1-\frac{b f}{c q}\right)}}{\left(1-\frac{(1-b)\left(1-\frac{b d}{c}\right)\left(1-\frac{b e}{c}\right)}{\left(1-\frac{b q}{f}\right)\left(1-\frac{b f}{c q}\right)\left(1-\frac{b d e}{c}\right)}\right)} \\
& \times\left\{{ }_{9} \phi_{8}\left[\begin{array}{cc}
q c^{\frac{1}{2}},-q c^{\frac{1}{2}}, b, b, c, d, e, f, \frac{c q^{2}}{f} & ; q, \frac{c}{b^{2} d e} \\
c^{\frac{1}{2}},-c^{\frac{1}{2}}, \frac{c q}{b}, q, \frac{c q}{d}, \frac{c q}{e}, \frac{c q}{f}, \frac{f}{q} &
\end{array}\right\} .\right.
\end{aligned}
$$

Similarly, other special cases can be derived by taking appropriate selections in the main results.

\section{Conclusions}

Certain results in the form of two-term, three-term and four-term transformation formulae had been discussed by the authors [12]. Certain new results in terms of basic hypergeometric series have been established in this paper. Our work extends the list of transformation formulae given in [12], that will provide some scope for future research in this field.

Acknowledgements. The authors express their sincere thanks to the editor and the anonymous reviewers for their valuable suggestions to revise this manuscript.

\section{REFERENCES}

[1] A. Schilling and S. Ole Warnaar, A higher-level Bailey lemma, Internat. J. Modern Phys. B 11 (1997), 189-195.

[2] A. Verma and V. K. Jain, Transformations between basic hypergeometric series on different bases and identities of Rogers-Ramanujan type, J. Math. Anal. Appl. 76(1) (1980), 230-269.

[3] A. Verma and V. K. Jain, Transformations of nonterminating basic hypergeometric series, their contour integrals and applications to Rogers-Ramanujan identities, J. Math. Anal. Appl. 87(1) (1982), 9-44.

[4] C. M. Joshi and Y. Vyas, Extensions of Bailey's transform and applications, Int. J. Math. Math. Sci. 12 (2005), 1909-1923.

[5] D. M. Bressoud, Some identities for terminating q-series, Math. Proc. Cambridge Philos. Soc. 89(2) (1981), 211-223.

[6] D. M. Bressoud, M. E. H. Ismail and D. Stanton, Change of base in Bailey pairs, Ramanujan J. 4(4) (2000), 435-453.

[7] G. E. Andrews, A general theory of identities of the Rogers-Ramanujan type, Bull. Amer. Math. Soc. 80 (1974), 1033-1052. 
[8] G. E. Andrews, An analytic generalization of the Rogers-Ramanujan identities for odd moduli, Proc. Natl. Acad. Sci. USA 71 (1974), 4082-4085.

[9] G. E. Andrews, Applications of basic hypergeometric functions, SIAM Review 16(4) (1974), 441-484.

[10] G. E. Andrews, Bailey's transform, lemma, chains and tree, Special functions 2000: current perspective and future directions, Tempe, AZ, NATO Sci. Ser. II Math. Phys. Chem., vol. 30, Kluwer Acad. Publ., Dordrecht, (2001), pp. 1-22.

[11] G. E. Andrews and S. Ole Warnaar, The Bailey transform and false theta functions, Ramanujan J. 14 (2007), 173-188.

[12] G. Gasper and M. Rahman, Basic Hypergeometric Series, Cambridge University Press, New York, 1991.

[13] H. S. Shukla, A note on the sums of certain bilateral hypergeometric series, Math. Proc. Cambridge Philos. Soc. 55(3) (1959), 262-266.

[14] I. Ali and V. K. Tuan, Application of basic hypergeometric series to stable analytic continuation, J. Comput. Appl. Math. 118(1-2) (2000), 193-202.

[15] L. J. Slater, A new proof of Rogers transformations of infinite series, Proc. Lond. Math. Soc. $\mathbf{5 3}(2)$ (1951), 460-475.

[16] L. J. Slater, Further identities of the Rogers-Ramanujan type, Proc. Lond. Math. Soc. 54(2) (1952), 147-167.

[17] M. Baker and D. D. Coon, Dual resonance theory with nonlinear trajectories, Phys. Rev. D 2 (1970), 2349-2358.

[18] N. G. Van Kampen, Exact calculation of the fluctuation spectrum for a nonlinear model system, J. Math. Phys. 2 (1961), 592-601.

[19] P. Srivastava, A note on Bailey's transform, South East Asian J. Math. Math. Sci. 2(2) (2004), 9-14.

[20] R. P. Agarwal, Resonance of Ramanujan's Mathematics, Vol. I, New Age International Pvt. Ltd. Publishers, New Delhi, 1996.

[21] R. Y. Denis, S. N. Singh and S. P. Singh, On Certain transformation formulae for abnormal q-series, South East Asian J. Math. Math. Sci. 1(3) (2003), 7-19.

[22] S. Ole Warnaar, 50 Years of Bailey's lemma, in Algebraic Combinatorics and Applications, pp. 333-347, A. Betten et al. eds., Springer, Berlin, 2001.

[23] S. P. Singh, Certain transformation formulae for q-series, Indian J. Pure Appl. Math. 31(10) (2000), 1369-1377.

[24] U. B. Singh, A note on a transformation of Bailey, Q. J. Math. 45(2) (1994), 111-116.

[25] V. K. Tuan, Stable analytic continuation using hypergeometric summation, Inverse Problems 15 (1999), 1-13.

[26] V. K. Tuan and M. Z. Nashed, Stable recovery of analytic functions using basic hypergeometric series, J. Comput. Anal. Appl. 3 (2001), 33-51.

[27] W. N. Bailey, Some identities in combinatory analysis, Proc. Lond. Math. Soc. 49(2) (1947), 421-425.

[28] W. N. Bailey, Identities of Rogers-Ramanujan type, Proc. Lond. Math. Soc. 50(2) (1949), 1-10. 


\author{
${ }^{1}$ Department of Mathematics \\ University of Petroleum and Energy Studies \\ Bidholi (P.O.) Via Prem Nagar \\ DEHRADUN-248007 \\ INDIA \\ E-mail address: mpathak81@rediffmail.com \\ ${ }^{2}$ Department of Mathematics \\ Motilal Nehru National Institute of Technology \\ AllahaBAD-211004 \\ INDIA \\ E-mail address: pankajs23@rediffmail.com
}

